Recebido em 03/2019. Aceito para publicação em 08/2019.

\title{
IMPLICAÇÕES SOCIOECONÔMICAS E A BIOSOCIODEMOGRAFIA DE UM CONJUNTO DE CRIANÇAS COM PARALISIA CEREBRAL EM URUGUAIANA
}

\section{SOCIOECONOMIC IMPLICATIONS AND BIOSOCIODEMOGRAPHY OF A SET OF CHILDREN WITH CEREBRAL PALSY IN URUGUAIANA}

\author{
Christian Caldeira Santos ${ }^{1}$ \\ Jacinta Sidegum Renner ${ }^{2}$
}

Resumo: Uruguaiana possui baixos indicadores sociais que podem influenciar a vida de crianças com paralisia cerebral. Objetivo desse artigo é contextualizar o perfil biosociodemográfico de crianças com paralisia cerebral que são assistidas por dois centros destinados a reabilitação física infantil em Uruguaiana. Foi utilizado como método a pesquisa aplicada, descritiva e de estudo de caso. A amostra foi de crianças com diagnóstico clínico de paralisia cerebral, de quatro a doze anos, que realizavam tratamento fisioterapêutico nos dois centros de reabilitação e residentes em Uruguaiana no mínimo há um ano, e seus pais/responsáveis. O perfil biosociodemográfico foi avaliado por um questionário estruturado e pelo Critério de Classificação Econômica Brasil (2018) respondidos pelos pais/responsáveis, assim como o Sistema de Classificação da Função Motora Grossa aplicado nas crianças. A análise dos dados foi realizada por meio da estatística descritiva e os resultados obtidos destacam maior frequência do perfil de crianças com quadriplegia espástica, com altos níveis de comprometimento motor, do sexo masculino, de menores classes sociais, que recebem benefício governamental, que residem em moradias próprias, em bairros periféricos e com benfeitorias básicas residenciais. A pesquisa concluiu que fatores do cenário regional associado aos do perfil biosociodemográfico geram um status quo de vulnerabilidade e fomenta a exclusão social nesse grupo de criança com paralisia cerebral e de seus familiares.

Palavra-chaves: Crianças; paralisia cerebral; perfil biosociodemográfico; indicadores sociais.

Abstract: Uruguaiana has low social indicators that can influence the life of children with cerebral palsy. Objective: to contextualize the biosociodemographic profile of children with cerebral palsy who are assisted by two centers for the physical rehabilitation of children in Uruguaiana. Method: Applied, descriptive and case study research. The sample was of children with clinical diagnosis of cerebral palsy, from four to twelve years old, who underwent physiotherapeutic treatment in the two rehabilitation centers and were residents of Uruguaiana for at least one year, and their parents / guardians. The biosociodemographic profile was evaluated by a structured questionnaire and the Brazilian Economic Classification Criterion (2018) answered by the parents / guardians, as well as the Gross Motor Function Classification System applied in the children. Data analysis was performed using descriptive statistics. Results: the profile presents a higher frequency of children with spastic quadriplegia, with high levels of motor impairment, male, of lower social classes, receiving government benefits, living in their own homes, in peripheral neighborhoods and with basic residential improvements. Final considerations: factors of the regional scenario associated with the biosociodemographic profile generate a status quo of vulnerability and promote social exclusion in this group of children with cerebral palsy and their relatives.

Keywords: Children; cerebral palsy; biosociodemographic profile; social indicators.

\section{INTRODUÇÃO}

No Brasil, o Bioma Pampa é exclusivo do estado do Rio Grande do Sul (RS) e

\footnotetext{
1 Docente do curso de Fisioterapia da Universidade Federal do Pampa, Campus Uruguaiana., Brasil. E-mail: christiansantos@unipampa.edu.br.

2 Doutora e Mestre em Engenharia de Produção com ênfase em Ergonomia (UFRGS), Docente da Universidade Feevale. Brasil. E-mail: jacinta@feevale.br.
} 
ocupa $63 \%$ do seu território, principalmente na sua metade Sul, estendendo-se até os territórios uruguaios e argentinos (INSTITUTO BRASILEIRO DE FLORESTA, 2017). Segundo Cargnin (2014), a região pampeana do RS concentra suas atividades na pecuária extensiva, fruticultura, silvicultura e orizicultura. Fonseca (2013) cita que este contexto geográfico configura um cenário de menor aporte empregatício e de baixos indicadores sociais, principalmente, aos relacionados à educação e à saúde.

Quanto aos quesitos indicadores sociais, a Fundação de Economia e Estatística (FEE) do RS mensura a situação socioeconômica dos municípios gaúchos em relação à educação, à renda e à saúde, levando em consideração aspectos quantitativos e qualitativos do processo de desenvolvimento. Os resultados são classificados em: Índice de Desenvolvimento Socioeconômico (IDESE) alto (maior de 0,800), médio (entre 0,500 e 0,799) e baixo (menor de 0,499) (RIO GRANDE DO SUL, 2019).

Assim, mais ao Sudoeste do estado situa-se a maior cidade da região, Uruguaiana. Seu IDESE de 2015 apontou uma classificação média $(0,670)$ de desenvolvimento, que a fez ocupar a 442 $2^{\text {a }}$ posição entre os 497 municípios gaúchos. Esse Índice é resultante da soma de três blocos de indicadores: Saúde $(0,714)$, Educação $(0,702)$ e Renda $(0,594)$ (FUNDAÇÃO..., 2019).

Em 2018 foi estimada uma população uruguaianense de 127.079 habitantes, ocupando a posição 14 cidade mais populosa do estado. Entretanto, em 2016 seu Produto Interno Bruto per capita a preços correntes foi de $R \$ 20.522,98$, que levou a cidade a ocupar a $408^{a}$ posição. No mesmo ano, o salário médio mensal dos uruguaianenses foi de 2,2 salários mínimos. A proporção de munícipes ocupados em relação à população total foi de $17 \%$. Para estes dados, Uruguaiana ocupou a posição

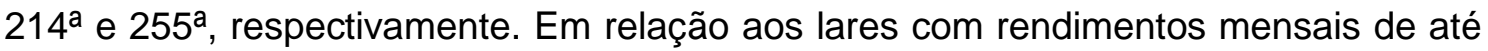
meio salário mínimo por pessoa, a cidade apresentou $35,6 \%$ da população nessas condições, o que lhe atribuiu a 119a posição (INSTITUTO BRASILEIRO DE GEOGRAFIA E ESTATíSTICA, 2019). Nessa tendência, Martins e Wink Junior (2014) destacaram que a região uruguaianense é marcada por uma alta concentração de pessoas em condições de extrema pobreza. Na tentativa minimização dessa condição regional, o Programa Bolsa Família beneficiou 5.386 famílias em Uruguaiana no ano de 2018 (BRASIL, 2019). E no quesito assistência à saúde, Uruguaiana apresentou 89,69\% de sua população dependente do Sistema Único de Saúde (SUS) (BRASIL, 2019).

Assim, neste cenário socioeconômico e de assistência à saúde estão presentes as crianças com paralisia cerebral (PC). Segundo o Ministério da Saúde, uma maior prevalência de casos de PC está relacionada com o pior suporte no período pré-natal e à qualidade do atendimento primário às gestantes, características estas que são comuns nos países em desenvolvimento,(BRASIL, 2014). Neste caso, Uruguaiana apresentou o índice de consultas de pré-natal menor do que o parâmetro estabelecido que é de 90\% pelo Ministério da Saúde (RIO GRANDE DO SUL, 2016).

De certo, estas crianças apresentam lesão encefálica que acarretou em distúrbios 
motores. Estes distúrbios podem vir associados, ou, não à perturbação sensorial e cognitiva (PATO et al., 2002). De praxe, a topografia da lesão da PC relaciona-se com a região do acometimento corporal, uni (hemiplegia) ou bilateralmente (diplegia ou quadriplegia) e o tipo de clínico está relacionado com o tônus muscular presente nessas regiões corporais acometidas (espástico, hipotônico, atetóide, ataxico ou misto).

Embora a PC ocorra em crianças pertencentes às famílias de todas as classes socioeconômicas, visualiza-se em pesquisas brasileiras (CORREIA; SALIMENE, 2016; ZAMPIERI; SANTOS; PFEIFER, 2016; NEVES; PIETROVSKI; CLAUDINO, 2015; TOLEDO et al., 2015; RIBEIRO et al., 2014; ALMEIDA et al., 2013; OLIVEIRA; MATSUKURA, 2013; ALCASSA et al., 2013; ASSIS-MADEIRA; CARVALHO; BLASCOVI-ASSIS, 2013) que ela é mais frequente nas mais desprovidas financeiramente.

No tensionamento entre fator socioeconômico e deficiência, o estudo de AssisMadeira, Carvalho e Blascovi-Assis (2013) sugeriu que o nível socioeconômico familiar possa exercer influência na gravidade do quadro e no desenvolvimento da criança com PC. Outro estudo apresentou que o desempenho funcional de pessoas com PC foi influenciado pelo ambiente social em que elas se encontravam (MANCINI et al., 2004). Já no texto de Baladi, Castro e Moraes Filho (2007) foi descrito que o fator socioeconômico familiar é um obstáculo para um prognóstico da criança com PC. Dessa forma, acredita-se que a condição socioeconômica familiar seja uma variável importante a ser considerada para a evolução sensório motora da criança com PC (REBEL et al., 2010).

Nesta vertente, possuindo Uruguaiana uma população com $6 \%$ de pessoas com deficiência motora (INSTITUTO BRASILEIRO DE GEOGRAFIA E ESTATÍSTICA, 2010), associado ao fato de Malheiros et al. (2013) demonstrarem que dentre as causas da deficiência física relacionadas ao período pré e perinatal, a PC apresentou ser mais frequente $(68 \%)$, e que "a pobreza é tanto uma causa como efeito da deficiência" (UNMIT, 2011, p. 15) no ser humano, surge um questionamento: qual o perfil biosociodemográfico de crianças com PC que usufruem de dois serviços destinados à reabilitação física infantil no município?

Com a perspectiva de responder este questionamento, aponta-se como objetivo deste estudo, contextualizar o perfil biosociodemográfico de crianças com PC que são assistidas por dois centros destinados à reabilitação física infantil em Uruguaiana.

\section{DESENVOLVIMENTO}

\subsection{Materiais e Método}

Este estudo integra uma pesquisa de âmbito mais abrangente que está focada em avaliar o estado de saúde, a qualidade de vida e o processo de inclusão social de 
crianças com PC no Pampa gaúcho.

De acordo com Prodanov e Freitas (2013), esta pesquisa é caracterizada em relação à sua natureza como pesquisa aplicada. Em relação a seus objetivos é classificada como descritiva e em relação aos procedimentos técnicos, ela atende pelo cunho de ser um estudo de caso, com análise de dados sob o paradigma quantitativo.

O estudo foi composto por crianças com diagnóstico clínico de PC e que frequentam dois centros de reabilitação física infantil de Uruguaiana. Ele apresenta amostragem não probabilística, do tipo por conveniência, visto que estes dois locais são referência municipal para o tratamento fisioterapêutico de crianças com PC.

Os critérios de inclusão elegidos para a pesquisa foram: as crianças com diagnóstico clínico de PC, com idades cronológicas entre 4 a 12 anos, que usufruem dos serviços de fisioterapia dos dois centros de reabilitação, residentes em Uruguaiana no período mínimo de um ano e seus respectivos pais/responsáveis. Acredita-se que neste tempo de residência, a criança e sua família já se encontram inter-relacionadas ao contexto ambiental, à rotina diária e aos serviços de saúde oferecidos pelo município. Esta afirmação é fundamentada na Teoria Bioecológica do Desenvolvimento de Urie Bronfenbrenner, onde essas crianças já oferecem e recebem influências do microssistema, mesossistema, exossistema, macrossistema, dentro de uma dimensão temporal (cronossistema) (PAPALIA; OLDS, 2000).

Os critérios de exclusão foram crianças com PC que sofreram intervenção médica invasiva nos últimos seis meses, e aquelas que possuírem pais/responsáveis com dificuldade de responder ao questionário biosociodemográfico.

Quanto aos procedimentos éticos, as crianças tiveram seus pais/responsáveis contactados pessoalmente pelo pesquisador, sendo que foram convidados juntamente com seu/sua filho(a) a participarem desta pesquisa. Os que aceitaram foram convidados a assinarem o Termo de Consentimento Livre e Esclarecido.

Para contextualizar o perfil biosociodemográfico das crianças com PC e de suas famílias, foi aplicado um questionário estruturado aos seus pais/responsáveis que continha perguntas relacionadas às questões biológicas, sociais e demográficas da criança e de sua família. Dois itens do questionário apresentam metodologias próprias de aplicação: a Gross Motor Function Classification System - Expanded and Revised (GMFCS - E \& R) e o Critério de Classificação Econômica - Critério Brasil 2018, da Associação Brasileira de Empresa de Pesquisa - ABEP (ASSOCIAÇÃO..., 2018).

O GMFCS - E \& R (Sistema de Classificação da Função Motora Grossa Expandido e Revisado) foi realizado por meio da observação direta da pessoa com PC, onde se determina o seu nível de comprometimento motor, de acordo com sua idade (0 - 18 anos). Ele é dividido em cinco níveis ${ }^{3}$. Quanto maior for o nível, pior será o

${ }^{3}$ Características gerais: Nível I - andam sem limitações; Nível II - andam com limitações; Nível III - andam utilizando um dispositivo manual de mobilidade; Nível IV - automobilidade com limitações e pode utilizar mobilidade motorizada; e 
desempenho motor da pessoa.

O Critério de Classificação Econômica da ABEP - Critério Brasil 2018, classifica as famílias brasileiras em classe social A, B1, B2, C1, C2, D e E, conforme com a pontuação adquirida pela quantidade de itens de conforto presentes no domicílio, pelo grau de instrução do chefe familiar e do acesso a serviços públicos (ASSOCIAÇÃO..., 2018).

As coletas de dados ocorreram durante os meses de agosto, novembro e dezembro de 2018 e fevereiro de 2019, nos dias em que era realizado o tratamento fisioterapêutico das crianças e nas dependências dos setores de reabilitação física da cidade. A análise desses dados foi realizada por meio de estatística descritiva, onde os resultados foram agrupados em dados nominais e ordinais.

A pesquisa foi aprovada pelo Comitê de Ética em Pesquisa da Universidade Feevale, com CAAE número 98591218.3.0000.5348, em 17 de outubro de 2018, pelo parecer número 2965.260, conforme determina a Resolução 466/12 do Conselho Nacional de Saúde que trata dos aspectos éticos em pesquisa envolvendo seres humanos no Brasil (BRASIL, 2012).

\subsection{Resultado e Discussão}

$\mathrm{Na}$ totalidade deste estudo, foram selecionadas 16 crianças de um universo de 26 crianças com PC assistidas pelos dois centros de reabilitação física infantil de Uruguaiana. Este conjunto de crianças com PC apresentou média de idade de 8,9 \pm 2,41 anos, sendo que 14 são naturais de Uruguaiana, ou seja, experimentam o contexto bioecológico desde o nascimento. Todas crianças estavam matriculadas em escolas regulares no período do estudo, conforme aponta a Lei 13.146 - Lei Brasileira de Inclusão da Pessoa com Deficiência (BRASIL, 2015).

A contextualização do perfil biosociodemográfico dessas crianças está apresentado conforme a Tabela 1. As crianças foram identificadas por nomes de deuses da mitologia grega, para assim, preservar suas identidades.

Tabela 1 - Perfil biosociodemográfico de crianças com paralisia cerebral de dois centros de reabilitação física infantil de Uruguaiana.

\begin{tabular}{ccccccc}
\hline $\begin{array}{c}\text { Criança, } \\
\text { tipo } \\
\text { topográfico, } \\
\text { clínico e } \\
\text { GMFCS E \& }\end{array}$ & $\begin{array}{c}\text { Problemas } \\
\text { relacionados }\end{array}$ & $\begin{array}{c}\text { Estrato } \\
\text { socioeconômico } \\
\text { ABEP }\end{array}$ & $\begin{array}{c}\text { Assistência } \\
\text { governamental }\end{array}$ & $\begin{array}{c}\text { Propriedade } \\
\text { da } \\
\text { residência }\end{array}$ & $\begin{array}{c}\text { Região da } \\
\text { residência }\end{array}$ & $\begin{array}{c}\text { Benfeitorias } \\
\text { presentes }\end{array}$ \\
\hline
\end{tabular}

Nível V - transportado em uma cadeira de rodas manual (PALISANO et al., 2007). 


\begin{tabular}{|c|c|c|c|c|c|c|}
\hline $\begin{array}{c}\text { Édipo } \\
\text { Quadriplegia } \\
\text { Discinética } \\
\text { V }\end{array}$ & $\begin{array}{l}\text { Oftalmológico / } \\
\text { Osteomuscular / } \\
\text { Fala / Cognitivo }\end{array}$ & A & Não & $\begin{array}{l}\text { Moradia } \\
\text { própria }\end{array}$ & $\begin{array}{c}\text { Zona } \\
\text { urbana } \\
\text { Bairro } \\
\text { Área } \\
\text { periférica }\end{array}$ & $\begin{array}{l}\mathrm{EE} / \mathrm{AP} / \mathrm{RE} \\
\text { / RA / C / TP }\end{array}$ \\
\hline $\begin{array}{l}\text { Hércules } \\
\text { Quadriplegia } \\
\text { Espástica } \\
\text { IV }\end{array}$ & Osteomuscular & B2 & Sim & $\begin{array}{l}\text { Moradia } \\
\text { própria }\end{array}$ & $\begin{array}{c}\text { Zona } \\
\text { urbana } \\
\text { Bairro } \\
\text { Área } \\
\text { central }\end{array}$ & $\begin{array}{c}\mathrm{EE} / \mathrm{AP} / \mathrm{RE} \\
/ \mathrm{RP} / \\
\mathrm{RA} / \mathrm{C} / \\
\mathrm{TP}\end{array}$ \\
\hline $\begin{array}{l}\text { Deméter } \\
\text { Hemiplegia } \\
\text { Espástica } \\
\text { I }\end{array}$ & $\begin{array}{l}\text { Osteomuscular / } \\
\text { Epilepsia }\end{array}$ & D & Não & $\begin{array}{l}\text { Moradia } \\
\text { própria }\end{array}$ & $\begin{array}{l}\text { Zona rural } \\
\text { Distrito }\end{array}$ & EE \\
\hline $\begin{array}{c}\text { Ajax } \\
\text { Quadriplegia } \\
\text { Espástica } \\
\text { V }\end{array}$ & $\begin{array}{l}\text { Oftalmológico / } \\
\text { Osteomuscular / } \\
\text { Fala / Cognitivo / } \\
\text { Epilepsia / } \\
\text { Gastroenterológico / } \\
\text { Pneumológico }\end{array}$ & B2 & Sim & $\begin{array}{l}\text { Moradia } \\
\text { alugada }\end{array}$ & $\begin{array}{c}\text { Zona } \\
\text { urbana } \\
\text { Bairro } \\
\text { Área } \\
\text { periférica }\end{array}$ & $\mathrm{EE} / \mathrm{AP} / \mathrm{TP}$ \\
\hline $\begin{array}{c}\text { Aquiles } \\
\text { Diplegia } \\
\text { Espástica } \\
\text { III }\end{array}$ & $\begin{array}{l}\text { Oftalmológico / } \\
\text { Osteomuscular / } \\
\text { Cognitivo }\end{array}$ & C1 & Sim & $\begin{array}{l}\text { Moradia } \\
\text { própria }\end{array}$ & $\begin{array}{c}\text { Zona } \\
\text { urbana } \\
\text { Bairro } \\
\text { Área } \\
\text { periférica }\end{array}$ & $\begin{array}{c}\mathrm{EE} / \mathrm{AP} / \mathrm{RE} \\
\text { / RP / RA / } \\
\mathrm{TP}\end{array}$ \\
\hline $\begin{array}{l}\text { Poseidon } \\
\text { Quadriplegia } \\
\text { Misto } \\
\text { V }\end{array}$ & $\begin{array}{l}\text { Otorrinolaringológico } \\
\text { / Osteomuscular / } \\
\text { Fala / Cognitivo / } \\
\text { Epilepsia / } \\
\text { Gastroenterológico }\end{array}$ & $\mathrm{C} 2$ & Sim & $\begin{array}{l}\text { Moradia } \\
\text { alugada }\end{array}$ & $\begin{array}{c}\text { Zona } \\
\text { urbana } \\
\text { Bairro } \\
\text { Área } \\
\text { periférica }\end{array}$ & $\begin{array}{l}\text { EE / AP / RE } \\
\text { / RP / RA/ C } \\
\text { / TP }\end{array}$ \\
\hline $\begin{array}{c}\text { Apolo } \\
\text { Quadriplegia } \\
\text { Hipotônica } \\
\text { IV }\end{array}$ & $\begin{array}{c}\text { Oftalmológico / } \\
\text { Otorrinolaringológico } \\
\text { / Osteomuscular / } \\
\text { Fala / Cognitivo / } \\
\text { Gastroenterológico }\end{array}$ & A & Não & $\begin{array}{l}\text { Moradia } \\
\text { própria }\end{array}$ & $\begin{array}{c}\text { Zona } \\
\text { urbana } \\
\text { Bairro } \\
\text { Área } \\
\text { central }\end{array}$ & $\begin{array}{l}\mathrm{EE} / \mathrm{AP} / \mathrm{RE} \\
\mathrm{RP} / \mathrm{RA} / \mathrm{C} / \\
\mathrm{TP}\end{array}$ \\
\hline $\begin{array}{c}\text { Hipnos } \\
\text { Quadriplegia } \\
\text { Espástica } \\
\text { V }\end{array}$ & $\begin{array}{c}\text { Oftalmológico / } \\
\text { Osteomuscular / } \\
\text { Fala / Epilepsia / } \\
\text { Cognitivo / } \\
\text { Gastroenterológico / } \\
\text { Pneumológico }\end{array}$ & B2 & Sim & $\begin{array}{l}\text { Moradia } \\
\text { própria }\end{array}$ & $\begin{array}{l}\text { Zona } \\
\text { urbana } \\
\text { Bairro } \\
\text { Área } \\
\text { periférica }\end{array}$ & $\begin{array}{c}\mathrm{EE} / \mathrm{AP} / \mathrm{RE} \\
\text { / RP / RA / C } \\
\text { / TP }\end{array}$ \\
\hline $\begin{array}{l}\text { Hefesto } \\
\text { Hemiplegia } \\
\text { Espástica } \\
\text { I }\end{array}$ & $\begin{array}{l}\text { Oftalmológico / } \\
\text { Osteomuscular / } \\
\text { Fala / Cognitivo }\end{array}$ & C1 & Sim & $\begin{array}{l}\text { Moradia } \\
\text { alugada }\end{array}$ & $\begin{array}{c}\text { Zona } \\
\text { urbana } \\
\text { Bairro } \\
\text { Área } \\
\text { periférica }\end{array}$ & $\begin{array}{l}\mathrm{EE} / \mathrm{AP} / \\
\mathrm{RE} / \mathrm{TP}\end{array}$ \\
\hline $\begin{array}{c}\text { Hera } \\
\text { Quadriplegia } \\
\text { Hipotônica } \\
\text { III }\end{array}$ & $\begin{array}{c}\text { Oftalmológico / } \\
\text { Osteomuscular / } \\
\text { Fala / Epilepsia / } \\
\text { Cognição / } \\
\text { Gastroenterológico }\end{array}$ & $\mathrm{C} 2$ & Sim & $\begin{array}{l}\text { Moradia } \\
\text { invadida }\end{array}$ & $\begin{array}{c}\text { Zona } \\
\text { urbana } \\
\text { Bairro } \\
\text { Área } \\
\text { periférica }\end{array}$ & $\begin{array}{c}\mathrm{EE} / \mathrm{AP} / \mathrm{RE} \\
/ \mathrm{TP}\end{array}$ \\
\hline $\begin{array}{c}\text { Atena } \\
\text { Quadriplegia } \\
\text { Espástica } \\
\text { IV }\end{array}$ & $\begin{array}{l}\text { Oftalmológico / } \\
\text { Osteomuscular / } \\
\text { Fala / Cognição / } \\
\text { Pneumológico }\end{array}$ & $\mathrm{C} 2$ & Sim & $\begin{array}{l}\text { Moradia } \\
\text { própria }\end{array}$ & $\begin{array}{c}\text { Zona } \\
\text { urbana } \\
\text { Bairro } \\
\text { Área } \\
\text { periférica }\end{array}$ & $\mathrm{EE} / \mathrm{AP} / \mathrm{TP}$ \\
\hline $\begin{array}{l}\text { Iris } \\
\text { Hemiplegia } \\
\text { Espástica } \\
\text { I }\end{array}$ & $\begin{array}{l}\text { Osteomuscular / } \\
\text { Fala }\end{array}$ & B2 & Não & $\begin{array}{l}\text { Moradia } \\
\text { própria }\end{array}$ & $\begin{array}{c}\text { Zona } \\
\text { urbana } \\
\text { Bairro } \\
\text { Área } \\
\text { central }\end{array}$ & $\begin{array}{c}\text { EE / AP / RE } \\
\text { / RP / RA / C } \\
\text { / TP }\end{array}$ \\
\hline
\end{tabular}




\begin{tabular}{|c|c|c|c|c|c|c|}
\hline $\begin{array}{c}\text { Hestia } \\
\text { Diplegia } \\
\text { Misto } \\
\text { III }\end{array}$ & Osteomuscular & $\mathrm{C} 1$ & Sim & $\begin{array}{l}\text { Moradia } \\
\text { alugada }\end{array}$ & $\begin{array}{c}\text { Zona } \\
\text { urbana } \\
\text { Bairro } \\
\text { Área } \\
\text { central }\end{array}$ & $\begin{array}{l}\mathrm{EE} / \mathrm{AP} / \mathrm{RE} \\
\text { / RP / RA/ C } \\
\text { / TP }\end{array}$ \\
\hline $\begin{array}{c}\text { Electra } \\
\text { Hemiplegia } \\
\text { Espástica } \\
\text { I }\end{array}$ & Osteomuscular & $A$ & Não & $\begin{array}{l}\text { Moradia } \\
\text { própria }\end{array}$ & $\begin{array}{c}\text { Zona } \\
\text { urbana } \\
\text { Bairro } \\
\text { Área } \\
\text { central }\end{array}$ & $\begin{array}{c}\mathrm{EE} / \mathrm{AP} / \mathrm{RE} \\
\text { / RP / RA / C } \\
\text { / TP }\end{array}$ \\
\hline $\begin{array}{c}\text { Elpis } \\
\text { Quadriplegia } \\
\text { Espástica } \\
\text { V }\end{array}$ & $\begin{array}{c}\text { Oftalmológico / } \\
\text { Osteomuscular / } \\
\text { Fala / Cognitivo / } \\
\text { Epilepsia / } \\
\text { Gastroenterológico / } \\
\text { Pneumológico }\end{array}$ & D & Sim & $\begin{array}{l}\text { Moradia } \\
\text { própria }\end{array}$ & $\begin{array}{c}\text { Zona } \\
\text { urbana } \\
\text { Bairro } \\
\text { Área } \\
\text { periférica }\end{array}$ & EE / AP / TP \\
\hline $\begin{array}{c}\text { Ártemis } \\
\text { Quadriplegia } \\
\text { Espástica } \\
\text { IV }\end{array}$ & $\begin{array}{l}\text { Osteomuscular / } \\
\text { Cognição }\end{array}$ & D & Sim & $\begin{array}{l}\text { Moradia } \\
\text { própria }\end{array}$ & $\begin{array}{c}\text { Zona } \\
\text { urbana } \\
\text { Bairro } \\
\text { Área } \\
\text { periférica }\end{array}$ & $\mathrm{EE} / \mathrm{AP} / \mathrm{TP}$ \\
\hline
\end{tabular}

Legenda: EE - Energia Elétrica; AP - Água Potável; RE - Rede de Esgoto; RP - Rede Pluvial; RA - Rua Asfaltada; C - Calçada; TP - Transporte Público.

Fonte: Autores da pesquisa.

Quanto ao tipo clínico e topográfico da PC houve uma frequência maior para o tipo espástico $(n=11)$ e da quadriplegia $(n=10)$ respectivamente, aonde este resultado vem ao encontro do que é sustentado por Baladi, Castro e Morais Filho (2007), pois informam que este tipo é presente em $75 \%$ e a quadriplegia em até $45 \%$ dos episódios de PC.

$\mathrm{Na}$ análise do GMFCS - E \& R houve frequência maior de crianças com PC $(n=12)$ com níveis de comprometimentos motores mais avançados (III, IV e V), onde suas limitações funcionais são mais graves, culminando segundo Palisano et al. (2007) desde a ausência de deambulação com transporte em uma cadeira de rodas até o andar com uso de um dispositivo manual de mobilidade (bengalas, muletas e andadores). Infelizmente, o agravamento do nível da GMFCS - E \& R nas crianças com PC parece impactar no processo de inclusão social destas crianças ao longo do tempo, visto que Zanelato et al. (2014) descreveram que adultos com PC com nível menor ou igual a III de GMFCS obtiveram maior possibilidade de avançar nas etapas escolares, todavia, aqueles com o maior nível de GMFCS possuíram 69\% de chance de não serem alfabetizados. Já no campo ocupacional, adultos com PC de nível I de GMFCS obtiveram $31 \%$ de possibilidade de conseguir um emprego, enquanto os de nível $\mathrm{V}$ apresentaram 99\% de chance de não estarem empregados.

Quanto aos problemas associados, foi verificado que o problema osteomuscular é presente em todas as crianças, decorrentes da alteração do tônus muscular e da força muscular, assim como os desalinhamentos articulares que culminam em deformidades ósseas nos membros superiores, inferiores e tronco. Outro problema associado evidenciado foi o déficit cognitivo. Segundo Baladi, Castro e Morais Filho (2007) cerca 
de $1 / 4$ das crianças com PC apresentam a função cognitiva normal e que tais déficits apresentam dificuldades ou impossibilidades de se testar em decorrência da disfunção sensório-motor grave e/ou da afecção da fala. Para estes autores os problemas associados podem interferir na etapa de reabilitação e assim, essas crianças necessitam de uma equipe interdisciplinar para administrar possíveis soluções desses problemas.

Quanto à frequência do sexo no conjunto dessas crianças, foi apresentado frequência maior do sexo masculino ( $n=9)$, semelhante aos estudos de Zampiere (2016), Correia e Salimene (2016) e de Toledo et al. (2015). Já no estudo de Cavalcante et al. (2017) houve uma distribuição igualitária entre os sexos das 122 crianças com PC pesquisadas. Assim, nesse quesito não se pode afirmar que exista uma predominância masculina sobre a feminina na PC, embora Almeida, Rodrigues e Salgado (2012) relatarem, com certo zelo, que em situações de possível dano encefálico (prematuridade e baixo peso ao nascer) a população masculina está mais suscetível que a feminina. Isso foi constatado nas crianças com PC aqui contextualizadas ( $n=5$ meninos e $n=3$ meninas) que apresentaram a prematuridade como fator de risco quanto ao período perinatal. Sob esse aspecto, Uruguaiana possui taxa de prematuridade maior que $11,26 \%$, onde o valor referencial e ideal seria abaixo de 10\% (RIO GRANDE DO SUL, 2016).

Entende-se que a minimização desse fator de risco para a PC esteja atrelada ao acompanhamento gestacional na fase pré-natal. Para tanto, sabe-se que o indicador percentual de sete ou mais consultas de pré-natal de Uruguaiana está em torno de $69,5 \%$, sendo que o parâmetro é de 90\% (RIO GRANDE DO SUL, 2016). Segundo informações adquiridas todas as mães fizeram o acompanhamento do pré-natal de suas gestações, entretanto, não foi questionado o número de consultas realizadas.

Assim, visando o aumento de cobertura no pré-natal e, consequentemente, um melhor nível no IDESE geral e do sub-bloco saúde, o Plano Municipal de Saúde 2018 2021 de Uruguaiana traz uma diretriz - proporção da atenção integral à saúde da mulher e da criança e implantação da Rede Cegonha, com ênfase nas áreas e populações de maior vulnerabilidade - que objetiva organizar a Rede de Atenção à Saúde Materno Infantil para garantir acesso, acolhimento e resolutividade, por meio de ações com vista a ampliar a captação precoce de gestantes no primeiro trimestre gestacional; realizar busca ativa de gestantes ausentes de consultas pré-natais; e garantir exames básicos neste período (URUGUAIANA, 2017). Nesta vertente Melo, Oliveira e Mathias (2015) afirmaram que uma parcela de crianças nascidas prematuramente pode ser prevenida por meio do pré-natal de qualidade e supervisão intensa dos itens determinados pelo Ministério da Saúde e também por meio dos programas nacionais, estaduais. Assim, espera-se para o futuro que esta diretriz contribua não somente para o aumento de indicadores de saúde, mas também, mesmo que indiretamente, com a redução da prevalência de PC no município. 
$\mathrm{Na}$ estratificação socioeconômica pela ABEP das famílias de criança com PC foi averiguado uma situação heterogênea de classes sociais, onde somente a classe E não foi presença entre as famílias. Nove grupos familiares pertenceram às classes sociais mais carentes ( $C 1, C 2$ e $D$ ) e sete pertenceram às mais elevadas ( $A$ e B2), de modo que este resultado reforça a afirmação que a $P C$ é mais frequente nas famílias mais desprovidas financeiramente.

Ao relacionar a estratificação socioeconômica familiar com o nível de GMFCS E \& $\mathrm{R}$ das crianças com PC percebe-se uma variabilidade nos dados. Entretanto, ao se agrupar as crianças com PC de menor nível de GMFCS (I e II) e de maior nível (III, IV e $\mathrm{V})$ fica exposta uma frequência maior de crianças com maiores comprometimentos motores $(n=7)$ inseridas em contextos familiares de menores aportes socioeconômicos (Classes $\mathrm{C}$ e D). Tal realidade demonstra um somatório de vulnerabilidade social, onde a primeira se dá pela própria deficiência e a outra pelo fator socioeconômico. Segundo Baltor e Dupas (2013) estas duas condições repercutem como fonte de angustia e preocupação familiar, visto que o processo de habilitação e reabilitação infantil ser dispendioso financeiramente e o serviço público não corresponder às expectativas e às necessidades reais das crianças.

Ao todo, 11 famílias receberam assistência governamental federal oriunda do Benefício de Prestação Continuada (BPC). Este Benefício é ofertado à pessoa com deficiência de qualquer tipo, idade e que apresentarem impedimentos de longo prazo (mínimo de dois anos), onde, a renda per capita da família tem que ser menor de $1 / 4$ do salário mínimo nacional vigente no ano. Nesse conjunto de famílias, a média de pessoas foi de 4,3 $\pm 1,1$ e com base no salário mínimo vigente para o ano de 2019 , a renda per capita foi de $R \$ 232,09$. Assim, infere-se que essas crianças estão em situação de vulnerabilidade à pobreza, pois suas famílias possuem rendas domiciliares per capita menores do que $\mathrm{R} \$ 255,00$ (PROGRAMA DAS NAÇÕES UNIDAS PARA O DESENVOLVIMENTO, 2017). Este retrato socioeconômico ajudou a compor o valor do IDESE geral e do sub-bloco renda de Uruguaiana, tendo este sub-bloco o menor índice entre os três (Saúde, Educação e Renda).

Desta forma, afirma-se que o fator renda tencionado à necessidade do tratamento da criança com PC, eclode na família um estado de sofrimento, visto que os custos do tratamento são elevados e o serviço público não cumpre seu dever. A essas barreiras e sofrimentos são somados preconceitos e estigmas vivenciados por esta população marginalizada e também por suas famílias. $O$ danoso resultado desse somatório míngua as ações de cidadania e justiça social, acarretando um processo de exclusão social pelo descompasso do processo de inclusão social (BALTOR; DUPAS, 2013).

Outro fator que contribuiu para o valor atual do IDESE geral e do sub-bloco educação foi o fato da maioria dos cuidadores principais $(n=10)$ apresentarem escolaridade mínima do Ensino Médio completo e o fato de todas as crianças estão inclusas na escola. Do ponto de vista socioeducacional, quanto maior a escolaridade 
das pessoas que integram a família, maiores serão as oportunidades de escolarização dos filhos e do aumento cultural familiar (UNICEF, 2005).

Quanto à moradia, onze famílias de crianças com PC apresentaram moradias próprias, quatro moradias alugadas e uma invadida. A localização destas residências engloba a maioria a zona urbana $(n=15)$ de Uruguaiana, sendo que a maioria $(n=10)$ está inserida em bairros periféricos. Existem famílias que tem suas moradias situadas em ruas não asfaltadas, o que por consequência, culmina na ausência de calçadas/passeios e de rede pluvial, além de não destino adequado do esgoto residencial. Para Dantas et al. (2012) a escassez de infraestrutura de municípios e de lares é algo rotineiro nas vidas de crianças com PC. Todavia, esta situação não é exclusiva das crianças de Uruguaiana, visto que Barbosa (2016, p.144) afirma que as pessoas com deficiência de todo Brasil "encontram obstáculos que dificultam ou impossibilitam sua mobilidade, restringindo-as ao seu ambiente doméstico e cerceando sua independência, sua capacidade de ter uma vida produtiva e seu direito de ir e vir". Em continuidade, os serviços essenciais à vida estão ofertados a todas as famílias das crianças com PC de Uruguaiana, no caso a água potável e a energia elétrica. Já na vertente do transporte público, atualmente o serviço municipal passou por mudanças com a presença de ônibus novos e adaptados para o translado das pessoas com deficiência.

$\mathrm{Na}$ esfera do lazer desse conjunto de 16 crianças com PC de Uruguaiana foi apresentado por todos os pais/responsáveis resultados semelhantes aos de Messa et al. (2005), onde a praça pública demonstrou ser o principal espaço de lazer desse grupo de crianças e seus familiares. As autoras propõem ainda que o lazer é um sinalizador de qualidade de vida e um facilitador da inclusão social. Entretanto, em Uruguaiana as praças apresentam, no máximo, as calçadas rebaixadas e rampas de acesso a elas, não há praça que dispõe de brinquedos acessíveis ao público com deficiência.

Assim, a interação pessoa-ambiente proposto pelo modelo biopsicossocial da incapacidade despende da atenção minuciosa do contexto bioecológico, ao lidar com público com deficiência (OMS, 2013). Destaca-se que fatores ambientais, tanto físicos como sociais, contribuem para um impacto na funcionalidade destas crianças com PC de Uruguaiana, ou seja, ora são facilitadores (positivos), ora são inibidores (negativos).

\section{CONCLUSÃO}

A contextualização biosociodemográfica deste conjunto de 16 crianças com PC apontou que todas se encontram matriculadas no ensino escolar regular e a maioria é do sexo masculino. As crianças são pertencentes a classes sociais mais inferiores, que recebem o benefício social correspondente às pessoas com deficiência, moradores da zona urbana e de bairros periféricos do município de Uruguaiana, cuja principal forma de lazer está atrelada à utilização de praças públicas. Em termos 
de características clínicas da PC nas crianças há predominância da forma clínica espástica, do tipo topográfico quadriplégico, com níveis do GMFCS - E \& R mais limitantes funcionalmente e com problemas ortopédicos e musculares coadjuvantes.

De forma geral, o resultado deste estudo revela semelhanças aos de outros estudos com crianças com PC do território nacional, e reforça a díade: baixos indicadores sociais e deficiência humana.

Como limitações desse estudo, destaca-se primeiro: o fato de ser um estudo de caso, sendo que seus resultados não podem ser representativos para toda população com PC de Uruguaiana. Segundo: por ser uma parte de uma pesquisa maior, a qual envolve outros instrumentos de pesquisa validados e com metodologias próprias de avaliação, as crianças menores de quatro anos não puderam compor o grupo de crianças desta pesquisa, resultando nesse conjunto de 16 crianças.

\section{AGRADECIMENTO}

O presente trabalho foi realizado com apoio da Coordenação de Aperfeiçoamento de Pessoal de Nível Superior - Brasil (CAPES), pelo Programa Suporte à PósGraduação de Instituições de Ensino Particulares (PROSUC), Modalidade II.

\section{REFERÊNCIAS}

ASSOCIAÇÃO BRASILEIRA DE EMPRESAS DE PESQUISA - ABEP. Critério de Classificação Econômica Brasil, 2018. Disponível em: <http://www.abep.org/criteriobrasil>. Acesso em 21 ago. 2017.

ALCASSA, T. C. et al. Crianças tetraparéticas e cuidadores: caracterizando o perfil e a acessibilidade à tecnologia assistiva. Journal of Human Growth and Development, São Paulo, v. 23, n. 1, p. 107-111, jun. 2013.

ALMEIDA, C.; RODRIGUES, O.; SALGADO, M. Diferenças no desenvolvimento de meninos e meninas em condições de risco. Boletim de Psicologia, São Paulo, v. 62, n. 136, p. 1-14, jun. 2012.

ALMEIDA, K. M. et al. Fatores que influenciam a qualidade de vida de cuidadores de paralisados cerebrais. Fisioterapia em Movimento, Curitiba, v. 26, n. 2, p. 307-14, jun. 2013.

ASSIS-MADEIRA, E. A.; CARVALHO, S. G; BLASCOVI-ASSIS, S. M. Desempenho funcional de crianças com paralisia cerebral de níveis socioeconômicos alto e baixo. Revista Paulista de Pediatria, São Paulo, v. 31, n. 1, p. 51-57, mar. 2013.

BALADI, A. B. P. C.; CASTRO, N. M. D. de; MORAIS FILHO, M. C. Paralisia Cerebral. In: Fernandez, A. C.; Ramos, A. C. R.; Casalis, M. E. P.; Hebert, S. K. AACD: Medicina e Reabilitação Princípios e Prática. São Paulo: Artes Médicas, 2007. p. 1534. 
BALTOR, M. R. R.; DUPAS, G. Experiências de famílias de crianças com paralisia cerebral em contexto de vulnerabilidade social. Revista Latino-Americana de Enfermagem, Ribeirão Preto, v. 21, n. 4, p. 1-8, jul./ago. 2013.

BARBOSA, A. S. Mobilidade urbana para pessoas com deficiência no Brasil: um estudo em blogs. Urbe - Revista Brasileira de Gestão Urbana, Curitiba, v. 8, n. 1, p. 142-154, abr. 2016.

BRASIL. Lei no 13.146, de 6 de julho de 2015. Institui a Lei Brasileira de Inclusão da Pessoa com Deficiência (Estatuto da Pessoa com Deficiência). Diário Oficial da União, Brasília, 2015. Disponível em:

<http://www.planalto.gov.br/CCIVIL_03/_Ato2015-2018/2015/Lei/L13146.htm>. Acesso em: 11 nov. 2017.

BRASIL. Ministério da Saúde. Conselho Nacional de Saúde. Comissão Nacional de Ética em Pesquisa. Resolução no 466, de 12 de dezembro de 2012. Diário Oficail da União, Brasília, 2012. Disponível em:

<http://conselho.saude.gov.br/resolucoes/2012/Reso466.pdf>. Acesso em: 16 mar. 2016.

BRASIL. Ministério da Saúde. Secretaria de Atenção à Saúde. Departamento de Ações Programáticas Estratégicas. Diretrizes de atenção à pessoa com paralisia cerebral. Ministério da Saúde, Secretaria de Atenção à Saúde, Departamento de Ações Programáticas Estratégicas - Brasília: Ministério da Saúde, 2014.

BRASIL, Ministério da Saúde. Secretaria de Atenção à Saúde. Departamento de Atenção Básica. Nota Técnica. Assunto: Informações sobre as ações e programas do Departamento de Atenção Básica. Município: Uruguaiana, RS. Brasília, 2019. Disponível em: <http://dab2.saude.gov.br/sistemas/notatecnica/frmListaMunic.php>. Acesso em: 1 abr. 2019.

CARGNIN, A. P. Política nacional de desenvolvimento regional e repercussões no Rio Grande do Sul. Mercator (Fortaleza), Fortaleza, v.13, n.1, p.19-35, abr. 2014.

CAVALCANTE, V. M. V. et al. Perfil epidemiológico das crianças com paralisia cerebral em atendimento ambulatorial. Revista Enfermagem UERJ, Rio de Janeiro, v. 25, p. e8780, abr. 2017.

CORREIA, V. D.; SALIMENE, A. C. M. Perfil social de crianças e jovens com paralisia cerebral em um centro de reabilitação física. Acta Fisiátrica, São Paulo, v. 23, n. 1, p. 16-19, 2016.

DANTAS, M. S. de A. et al. Facilidades e dificuldades da família no cuidado à criança com paralisia cerebral. Rev. Gaúcha Enferm., Porto Alegre, v. 33, n. 3, p. 73-80, set. 2012.

FUNDAÇÃO DE ECONOMIA E ESTATÍSTICA DO RIO GRANDE DO SUL - FEE. Indicadores / Idese / Série Histórica Nova Metodologia / Índice de Desenvolvimento Socioeconômico (Idese) dos municípios do Rio Grande do Sul - 2015 / Uruguaiana. Disponível em: <https://www.fee.rs.gov.br/indicadores/indice-dedesenvolvimento-socioeconomico/serie-historica-novametodologia/?ano=2015\&letra=U\&ordem=municipios $>$. Acesso em: 6 maio 2019.

FONSECA, L. R. da S. C. M. Sociedade civil, esfera pública e hegemonia: um estudo sobre a criação da Universidade Federal do Pampa - UNIPAMPA. 2013. Tese 
(Doutorado em Serviço Social) - Pontifícia Universidade Católica do Rio Grande do Sul, Porto Alegre, 2013.

INSTITUTO BRASILEIRO DE FLORESTA - IBF. Bioma Pampa. Disponível em <https://www.ibflorestas.org.br/bioma-pampa.html>. Acesso em: 11 nov. 2017.

INSTITUTO BRASILEIRO DE GEOGRAFIA E ESTATÍSTICA - IBGE. Brasil/Rio Grande do Sul/ Uruguaiana/Panorama. Disponível em:

<https://cidades.ibge.gov.br/brasil/rs/uruguaiana/panorama>. Acesso em: 20 fev. 2019.

INSTITUTO BRASILEIRO DE GEOGRAFIA E ESTATÍSTICA - IBGE. Censo

Demográfico 2010 - Rio Grande do Sul. Rio de Janeiro, 2010. Disponível em <http://www.ibge.gov.br/estadosat/perfil.php?sigla=rs>. Acesso em: 26 jul. 2017.

MALHEIROS, R. T. et al. Perfil demográfico e socioeconômico das pessoas com deficiência física residentes na zona urbana da cidade de Uruguaiana - RS. Vittalle, Rio Grande, v. 25, n. 2, p. 41-51. 2013.

MANCINI, M. C. et al. Gravidade da paralisia cerebral e desempenho funcional. Revista Brasileira de Fisioterapia, São Carlos, v. 8, n. 3, p. 253-260. 2004.

MARTINS, C. H.; WINK JUNIOR, M. V. Pobreza extrema no Rio Grande do Sul e sua relação com o Índice de Desenvolvimento Socioeconômico (Idese). In: PICHLER, W. A. (Org.) et al. Panorama socioeconômico e perspectivas para a economia gaúcha. Porto Alegre: FEE, 2014.

MELO, E. C.; OLIVEIRA, R. R. de.; MATHIAS, T. A. de F. Fatores associados à qualidade do pré-natal: uma abordagem ao nascimento prematuro. Revista da Escola de Enfermagem da USP, São Paulo, v. 49, n. 4, p. 540-549, ago. 2015.

MESSA, A. A. et al. Lazer familiar: um estudo sobre a percepção de pais de crianças com deficiência. Cadernos de Pós-Graduação em Distúrbios do Desenvolvimento, São Paulo, v. 5, p. 9-25. 2005.

NEVES, E. B.; PIETROVSKI, E. F.; CLAUDINO, R. F. Qualidade de vida e dor lombar em cuidadores primários de crianças com paralisia cerebral. Cadernos de Saúde Coletiva, Rio de Janeiro, v. 23, n. 1, p. 50-56, jan./mar. 2015.

OLIVEIRA, A. K. C; MATSUKURA, T. S. Estresse e apoio social em cuidadores de crianças com paralisia cerebral. Cadernos Brasileiros de Terapia Ocupacional UFSCar, São Carlos, v. 21, n. 3, p. 493-503. 2013.

OMS - Organização Mundial da Saúde. Como usar a CIF: Um manual prático para o uso da Classificação Internacional de Funcionalidade, Incapacidade e Saúde (CIF). Versão preliminar para discussão. Genebra: OMS. Outubro de 2013.

PALISANO, R. et al. GMFCS - E \& R: Sistema de Classificação da Função Motora Grossa, Ampliado e Revisto. CanChild Centre for Chilhood Disability Research, McMaster University, Tradução: SILVA, D. B. R., PFEIFER, L. I., FUNAYAMA, C. A. R. Programa de Pós-Graduação em Neurociências e Ciências do Comportamento Faculdade de Medicina de Ribeirão Preto, Universidade de São Paulo, 2007.

PAPALIA, D. E.; OLDS, S. W. Desenvolvimento Humano. 7. ed. Porto Alegre: Artes Médicas Sul, 2000. 
PATO, T. R. et al. Epidemiologia da paralisia cerebral. Acta Fisiátrica, São Paulo, v. 9, n. 2, p. 71-76. 2002.

PRODANOV, C. C.; FREITAS, E. C. de. Metodologia do trabalho científico:

métodos e técnicas de pesquisa e do trabalho acadêmico. 2. ed. Novo Hamburgo:

Feevale, 2013.

PROGRAMA DAS NACCÕES UNIDAS PARA O DESENVOLVIMENTO, INSTITUTO DE PESQUISA ECONÔMICA APLICADA e FUNDAÇÃO JOÃO PINHEIRO. Atlas do

Desenvolvimento Humano no Brasil. Disponível em:

<http://atlasbrasil.org.br/2013/pt/consulta>. Acesso em: 7 ago. 2017.

REBEL, M. F. et al. Prognóstico motor e perspectivas atuais na paralisia cerebral. Revista Brasileira de Crescimento e Desenvolvimento Humano, São Paulo, v. 20, n. 2, p. 342-350, ago. 2010.

RIBEIRO, M. F. M. et al. Estresse parental em mães de crianças e adolescentes com paralisia cerebral. Revista Latino-Americana de Enfermagem, Ribeirão Preto, v. 22, n. 3, p. 440-447, maio-jun. 2014.

RIO GRANDE DO SUL. Secretaria de Planejamento, Governança e Gestão. Atlas Socioeconômico Rio Grande do Sul. Indicadores Sociais. Índice de Desenvolvimento Estadual - IDESE, 2019. Disponível em:

$<$ https://atlassocioeconomico.rs.gov.br/indice-de-desenvolvimento-socioeconomiconovo-idese >. Acesso em: 6 abr. 2019.

RIO GRANDE DO SUL. Secretaria Estadual de Saúde. Plano Estadual de Saúde 2016-2019 do Rio Grande do Sul, 2016. Disponível em:

<http://www.saude.rs.gov.br/plano-estadual-de-saude>. Acesso em: 15 fev. 2019.

TOLEDO et al. Perfil epidemiológico de crianças diagnosticadas com paralisia cerebral atendidas no Centro de Reabilitação Lucy Montoro de São José dos Campos. Acta Fisiátrica, São Paulo, v. 22, n. 3, p. 118 - 122. 2015.

UNICEF - FUNDO DAS NAÇÕES UNIDAS PARA A INFÂNCIA. Índice de Desenvolvimento Infantil. In: Situação da Infância Brasileira 2006. Brasília, 2005. Disponível em: <https://www.unicef.org/brazil/pt/resources_10167.htm>. Acesso em: 14 mar. 2019.

UNMIT - UNITED NATIONS MISSION IN TIMOR - LESTE. Claro que podemos. Relatório sobre os direitos das pessoas com deficiência em Timor - Leste, 2011.

URUGUAIANA. Prefeitura. Secretaria Municipal de Saúde. Plano Municipal de Saúde 2018 - 2021. Dezembro de 2017. Disponível em: <http://www.saude.rs.gov.br/planoestadual-de-saude>. Acesso em: 15 fev. 2019.

ZAMPIERI, L. M.; SANTOS, J. L.; PFEIFER, L. I. Validade discriminante do protocolo de desempenho funcional e social de crianças com paralisia cerebral. Acta Fisiátrica, São Paulo, v. 23, n. 2, p. 66-72. 2016.

ZAMPIERI, L. M. Validade concorrente, sensibilidade e praticabilidade do protocolo de avaliação do desempenho funcional e participação social de crianças com paralisia cerebral baseado na classificação internacional de funcionalidade incapacidade e saúde - CIF. 2016. Tese (Doutorado em Neurologia) - Faculdade de Medicina de Ribeirão Preto/USP. Universidade de São Paulo, Ribeirão 
Preto, 2016.

ZANELATO, N. et al. Associação dos níveis de GMFCS com graus de escolaridade, tipo de marcha, empregabilidade e dor em adultos com paralisia cerebral. Acta Fisiátrica, São Paulo, v. 21, n. 4, p. 158-161, 2014. 